\title{
Erratum to: Leveraging Applications of Formal Methods, Verification and Validation (Part I)
}

\author{
Tiziana Margaria $^{1(凶)}$ and Bernhard Steffen ${ }^{2}$ \\ 1 Lero, Limerick, Ireland \\ tiziana.margaria@lero.ie \\ 2 TU Dortmund, Dortmund, Germany
}

\section{Erratum to: \\ T. Margaria and B. Steffen (Eds.) \\ Leveraging Applications of Formal Methods, \\ Verification and Validation (Part I) \\ DOI: 10.1007/978-3-319-47166-2}

In the initially published version of chapter 61 of Part II (Leveraging Applications of Formal Methods, Verification and Validation), two author names were erroneously omitted. This has been updated. Consequently, the Table of Contents and the Author Index have also been updated in this volume (LNCS 9952), Part I (Leveraging Applications of Formal Methods, Verification and Validation). 